\title{
Radiação ultravioleta (uv-c) na qualidade pós-colheita de frutos de lichia 'bengal'
}

Juliana Arruda Ramos, Flávia Aparecida de Carvalho Mariano-Nasser, Veridiana Zocoler Mendonça, Karina Aparecida Furlaneto, Giovanna Alencar Lundgren, Rogério Lopes Vieites

Universidade Estadual Paulista - UNESP. E-mail: ju.a.ramos@globo.com

\section{Resumo}

Objetivou-se avaliar a influência da radiação (UV-C) na conservação de lichias da cv. 'Bengal' frigoarmazenadas. Os frutos foram submetidos a exposição da luz ultravioleta em equipamento IRINOX (UV-C com $\lambda=254 \mathrm{~nm}$ ), acondicionados em bandeja de poliestireno expandido sem recobrimento e armazenados em câmara fria $(5 \pm 0,5$ C e $90 \pm 5 \%$ de UR) por quinze dias. As análises foram realizadas a cada três dias e avaliados: perda de massa, atividade respiratória, pH, sólidos solúveis, acidez titulável, índice de maturação e ácido ascórbico. $O$ experimento foi conduzido em delineamento inteiramente casualizado, em esquema fatorial e os dados foram submetidos à análise de variância e regressão polinomial $(p<0,05)$. A radiação UV-C não foi efetiva para diminuição de perda de massa. Os tratamentos de 20 e 30 minutos de exposição à radiação UV-C foram eficazes na manutenção da concentração de ácido ascórbico.

Palavras-chave: ácido ascórbico; conservação; Litchi chinensis Sonn.

\section{Ultraviolet radiation (uv-c) in post-harvest quality of lichia 'bengal' fruit}

\begin{abstract}
The objective of this study was to evaluate the influence of radiation (UV-C) on the conservation of litchi of cv. 'Bengal' refrigerated storage. The fruits were submitted to ultraviolet light exposure in IRINOX equipment (UV-C with $\lambda=254 \mathrm{~nm}$ ), packaged in expanded polystyrene tray without overlay and stored in a cold room ( $5 \pm 0.5 \circ \mathrm{C}$ and $90 \pm 5 \%$ UR) for fifteen days. The analyzes were performed every three days and evaluated: loss of mass, respiratory activity, $\mathrm{pH}$, soluble solids, titratable acidity, maturation index and ascorbic acid. The experiment was conducted in a completely randomized design in a factorial scheme and the data were submitted to analysis of variance and polynomial regression $(p<0.05)$. UV radiation was not effective in reducing mass loss. The treatments of 20 and 30 minutes exposure to UV-C radiation were effective in maintaining ascorbic acid concentration.
\end{abstract}

Keywords: ascorbic acid; conservation; Litchi chinensis Sonn.

\section{Introdução}

A lichia (Litchi chinensis Sonn.) é um fruto tropical da família Sapindaceae com origem na China. Apresenta casca vermelha brilhante e polpa translúcida com grande valor nutritivo e padrão não climatério. A cultivar mais conhecida no Brasil é a 'Bengal', frutos cordiformes, peso de $21 \mathrm{~g}$ em média, polpa firme (65\% do fruto) e com semente grande (LIMA et al., 2011; SOUZA et al.; 2010; LIMA et al., 2010; MOTTA, 2009; HUANG, 2002).
Os frutos possuem alto teor de açúcar, minerais como potássio, magnésio, fósforo, zinco e manganês em quantidades apreciáveis e vitaminas como vitamina $C$, riboflavina, niacina e tiamina. (MENZEL; WAITE, 2005; WALL, 2006). Porém as quantidades dos nutrientes variam de acordo com o local de plantio devido variações de clima, solo e tratamento da planta. Por exemplo, o ácido ascórbico varia com a intensidade da luz (LEE; KADER, 2000). Por ser rica em ácido ascórbico, ingerindo aproximadamente quinze frutas já alcançamos a recomendação diária 
média em adultos. Outra característica nutricional relevante é a presença de substancias fenólicas como as antocianinas, os flavonóides e taninos condensados; que são importantes antioxidantes que nosso corpo necessita (SARNIMANCHADO et al., 2000; JIANG et al., 2007). Muitos estudos têm demonstrado a importância desses fitoquímicos na prevenção de diabetes, doenças cardiovasculares e câncer (HASLER et al., 2004; MERKEN; BEECHER, 2000).

Quando armazenada em temperatura ambiente, os frutos se tornam muito perecíveis, perdendo sua qualidade em apenas dois dias, devido a doenças pós-colheita e escurecimento (DEL AGUILA et al., 2009; HUANG, 2002). A característica principal para decisão de compra dos consumidores da lichia é a coloração vermelha da epiderme, sendo ainda considerado o aspecto mais importante de qualidade do fruto. Contudo a casca apresenta escurecimento enzimático, prejudicando sua comercialização, mesmo apresentando pouca alteração na qualidade da polpa (LIMA et al., 2010).

O método mais utilizado na conservação pós-colheita de frutos é a refrigeração. No entanto, outros métodos físicos podem ser associados a este e resultar em melhorias na sua conservação. A radiação (UV-C) é considerada um tratamento físico que pode ser usada para o controle de doenças pós-colheita, pois utiliza como método de controle a deterioração, resultando em desinfecção superficial dos frutos, reduzindo portanto o crescimento microbiano (MARQUENIE et al., 2003; VICENTE et al., 2005; KHADEMI et al., 2013; SRICASTAVA; SHARNA, 2013), além de atrasarem a maturação de frutos (WILSON et al., 1994). Segundo Stevens et al. (2004), atrasa o amolecimento do fruto, fator determinante na vida pós-colheita. Lu et al. (1993) trabalhando com pêssegos obtiveram resultados positivos quando ao atraso de maturação dos frutos e redução da podridão durante o armazenamento e Campos et al. (2011) relataram efeitos positivos na radiação (UV-C) sobre a qualidade de tomates 'Pitenza'.

Diante disso objetivou-se avaliar a influência da radiação (UV-C) na conservação de lichias da cv. 'Bengal' frigoarmazenadas.

\section{Material e Métodos}

Foram utilizadas lichias da cv. 'Bengal', no estádio de maturação fisiológica (frutos de coloração vermelha uniforme) provenientes de pomar comercial localizado no município de
Mogi-Mirim - SP, cujas coordenadas geográficas são: Latitude -22 을 $25^{\prime} 39^{\prime \prime} \mathrm{S}$, Longitude -460 57' 28" O e $632 \mathrm{~m}$ de altitude.

Os frutos selecionados foram submetidos a exposição da luz ultravioleta (UV-C $\operatorname{com} \lambda=254$ $\mathrm{nm})$, sendo a distância dos frutos à fonte de luz de $20 \mathrm{~cm}$ e o período de exposição de 10, 20 e 30 minutos em equipamento IRINOX, Refrigerador e Congelador da marca AREX (modelo: n-HCM 51). Os frutos sem aplicação de radiação nãoionizante constituíram o tratamento controle. Os frutos foram acondicionados em bandeja de poliestireno expandido sem recobrimento, cada embalagem recebeu em média 10 frutos ( \pm 200 gramas) e armazenados em câmara fria a $5 \pm 0,5$ ㅇ C e $90 \pm 5 \%$ de UR por 15 dias. As análises foram realizadas a cada três dias e avaliados: perda de massa, atividade respiratória, $\mathrm{pH}$, sólidos solúveis, acidez titulável, índice de maturação e ácido ascórbico.

A perda de massa foi realizada através da pesagem das embalagens em balança OWLABOR, considerando a massa inicial de cada amostra e aquela obtida a cada intervalo de tempo de amostragem, segundo a equação:

$$
P M(\%)=\frac{\left(P_{i}-P_{j}\right)}{P_{i}} .100
$$

Onde: $P M$ é a perda de massa; $P_{i}$ é a massa inicial e $P_{\mathrm{j}}$ é a massa no período avaliado. Os resultados foram expressos em porcentagem.

A atividade respiratória foi determinada pela liberação de $\mathrm{CO}_{2}$ liberado, de acordo com metodologia adaptada de Bleinroth et al. (1976), utilizando-se para isso solução de hidróxido de bário saturado e solução de hidróxido de potássio $0,1 \mathrm{~N}$, para tanto foi utilizado a seguinte fórmula:

$$
\mathrm{TCO}_{2}=\frac{2,2\left(\mathrm{Vo}_{\mathrm{V}} \mathrm{V}_{1}\right) 10}{\mathrm{M} \mathrm{T}}
$$

Onde: $\mathrm{T} \mathrm{CO}_{2}=$ taxa de respiração $\left(\mathrm{mlCO}_{2} \mathrm{Kg}^{-1} \mathrm{~h}^{-1}\right)$; Vo = volume gasto de $\mathrm{HCl}$ para titulação de hidróxido de potássio - padrão antes da absorção de $\mathrm{CO}_{2}(\mathrm{ml}) ; \mathrm{V} 1$ = volume gasto de $\mathrm{HCl}$ para titulação de hidróxido de potássio após a absorção de $\mathrm{CO}_{2}$ da respiração $(\mathrm{ml}) ; \mathrm{M}=$ massa dos frutos $(\mathrm{Kg}) ; \mathrm{T}=$ tempo da respiração; $2,2=$ inerente ao equivalente de $\mathrm{CO}_{2}$ (44/2), multiplicado pela concentração do ácido clorídrico; 10 = ajuste para o total de hidróxido de potássio usado no experimento.

$\mathrm{O} \mathrm{pH}$ foi medido na polpa triturada dos frutos utilizando-se potenciômetro (Digital DMPH-2), conforme metodologia do Instituto Adolfo Lutz (BRASIL, 2008). Os sólidos solúveis 
foram determinados em leitura refratométrica em ${ }^{\circ}$ Brix, a $20^{\circ} \mathrm{C}$, com o refratômetro digital tipo Palette PR-32, marca ATAGO, conforme metodologia de IAL (BRASIL, 2008). A acidez titulável foi expressa em g de ácido málico $100 \mathrm{~g}^{-1}$ de polpa e realizada através da titulação de $10 \mathrm{~g}$ de polpa homogeneizada e diluída para $90 \mathrm{ml}$ de água destilada, com solução padronizada de hidróxido de sódio a 0,1 N, tendo como indicador o ponto de viragem de fenolftaleína, que se dá quando o potenciômetro atinge 8,1 (BRASIL, 2008). O índice de maturação (Ratio) foi obtido através da relação entre os sólidos solúveis e a acidez titulável.

O teor de ácido ascórbico foi determinado por titulação pelo método colorimétrico com 2,4-dinitrofenilhidrazina, segundo Instituto Adolfo Lutz (BRASIL, 2008). Os resultados foram expressos em $\mathrm{mg}$ de ácido ascórbico $100 \mathrm{~g}^{-1}$ de polpa.

0 experimento foi conduzido em delineamento inteiramente casualizado (D.I.C.), em esquema fatorial $(4 \times 6)$, composto por quatro tempo de exposição de UV-C e seis períodos de armazenamento. Para as avaliações destrutivas foram utilizadas três repetições e para as avaliações não-destrutivas (perda de massa fresca) foi utilizada uma por tratamento. Os dados foram submetidos a análise de variância e regressão polinomial $(p<0,05)$.

\section{Resultados e Discussão}

Para a perda de massa, houve aumento dos valores com o período de armazenamento, possivelmente pela perda de água devido a transpiração dos frutos. $O$ controle e o tratamento com 20 minutos de exposição UV-C apresentaram as menores perdas de massa até o décimo quinto dia de armazenamento, chegando ao final com 14,0 e $13,7 \%$, enquanto os demais tratamentos apresentaram perdas de 16,8\% (10 minutos de UV-C) e 16,5\% (30 minutos de UV-C) ao final do armazenamento. Resultados semelhantes foram citados por Daiuto et al. (2013), que em pesquisa com abacate 'Hass' e tempos de exposição ao UV-C observaram perda de massa linear crescente no período de armazenamento. Os autores ainda citam que a maior perda foi nos frutos expostos por 5 minutos de UV-C.

Segundo Chitarra e Chitarra (2005), perdas de massa entre 3 a $6 \%$ são capazes de causar perda da qualidade dos frutos armazenados. Diante disso as lichias do tratamento 10 e 30 minutos de UV-C teriam em média 3 dias de armazenamento, enquanto o controle e 10 minutos de UV-C chegariam até o quinto dia com $6 \%$ de perda. Podendo então afirmar que o tratamento de lichia 'Bengal' com UV-C não foi efetivo, uma vez que o melhor tempo de exposição não diferiu do controle.

Figura 1. Perda de massa fresca (\%) de lichias cv. Bengal submetidas a exposição a luz UV-C durante o armazenamento refrigerado $(5 \pm 0,5 \stackrel{\circ}{ } \mathrm{C}$ e $90 \pm 5 \%$ UR) por 15 dias.

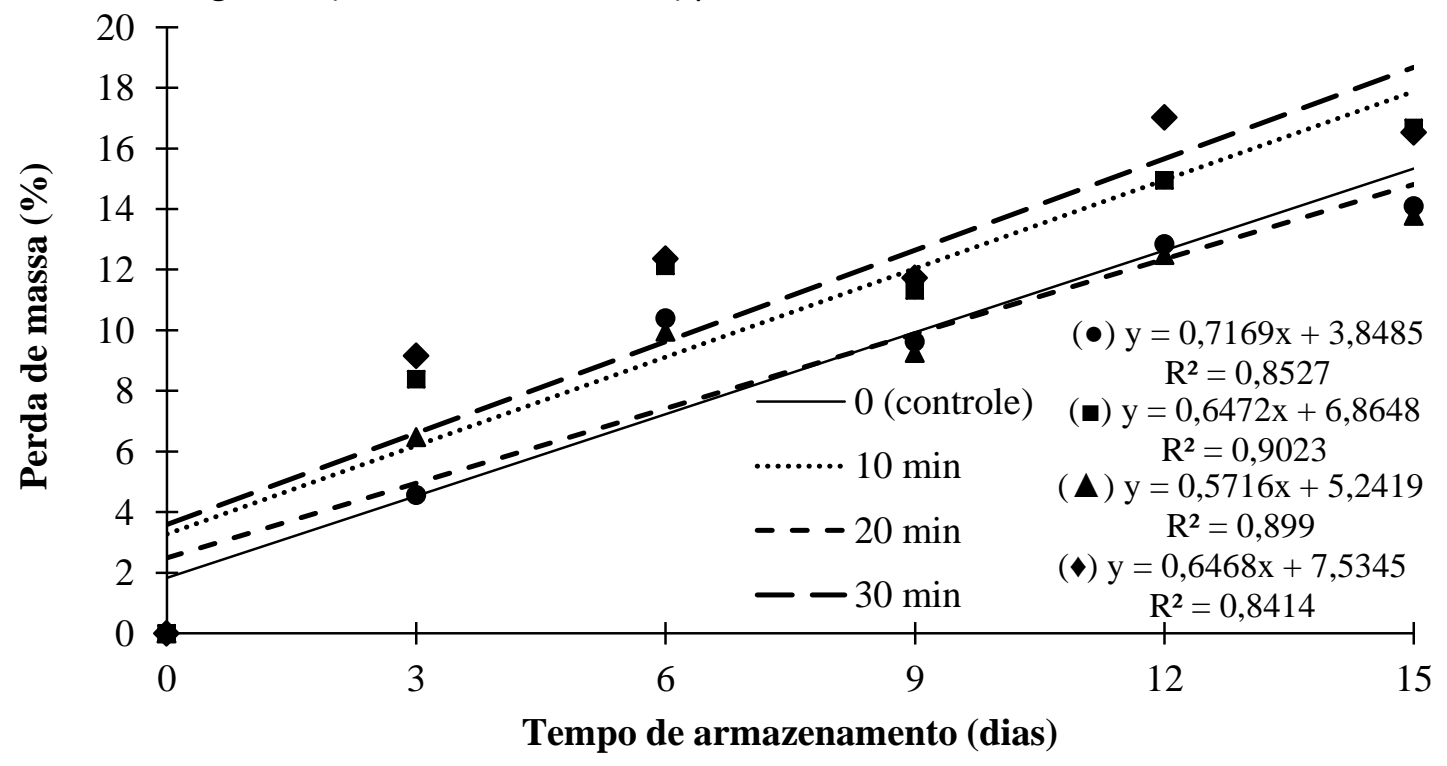

A atividade respiratória dos frutos caracteriza-se pelo $\mathrm{O}_{2}$ do ar que é absorvido e o $\mathrm{CO}_{2}$ liberado conduzindo a senescência do produto (NEVES, 2016). Nos frutos de lichia (Figura 2) observa-se redução da atividade respiratória com o avanço do período de 
armazenamento. A maior liberação de $\mathrm{CO}_{2}$ no dia 0 possivelmente está relacionada ao estresse causado pela colheita, transporte e manuseio (SOUZA; VIEITES; LIMA, 2010) que aumenta o metabolismo num primeiro momento (AGUILA et al., 2009). Entretanto, nas avaliações sequentes, observa-se redução da atividade respiratória e ausência de pico respiratório evidenciando o comportamento não climatérico dos frutos, assim como observado por Hojo et al. (2011).

Os frutos, testemunha e submetidos a radiação UV-C, apresentaram média respiratória geral de 24,89 $\mathrm{CO}_{2} \mathrm{~kg}^{-1} \mathrm{~h}^{-1}$ e os frutos com 10 minutos de exposição ao UV-C apresentaram, em média, a menor taxa respiratória ao longo dos 15 dias $\left(22,3 \mathrm{ml} \mathrm{CO} \mathrm{kg}^{-1} \mathrm{~h}^{-1}\right)$. Menores taxas respiratórias são indicativos positivos de conservação de frutos armazenados, pois se relaciona a redução metabólica, ou seja, menor consumo de reservas, aumentando, consequentemente, o período pós-colheita (SILVA et al., 2012), relacionando-se também a manutenção dos valores de pH obtidos (Figura 3) uma vez que os ácidos orgânicos são substratos respiratórios (SILVA et al., 2012).

Figura 2. Atividade respiratória $\left(\mathrm{ml} \mathrm{CO} \mathrm{kg}^{-1} \mathrm{~h}^{-1}\right.$ ) de lichias $\mathrm{cV}$. Bengal submetidas a exposição a luz UV-C durante o armazenamento refrigerado ( $5 \pm 0,5$ oC e $90 \pm 5 \%$ UR) por 15 dias.

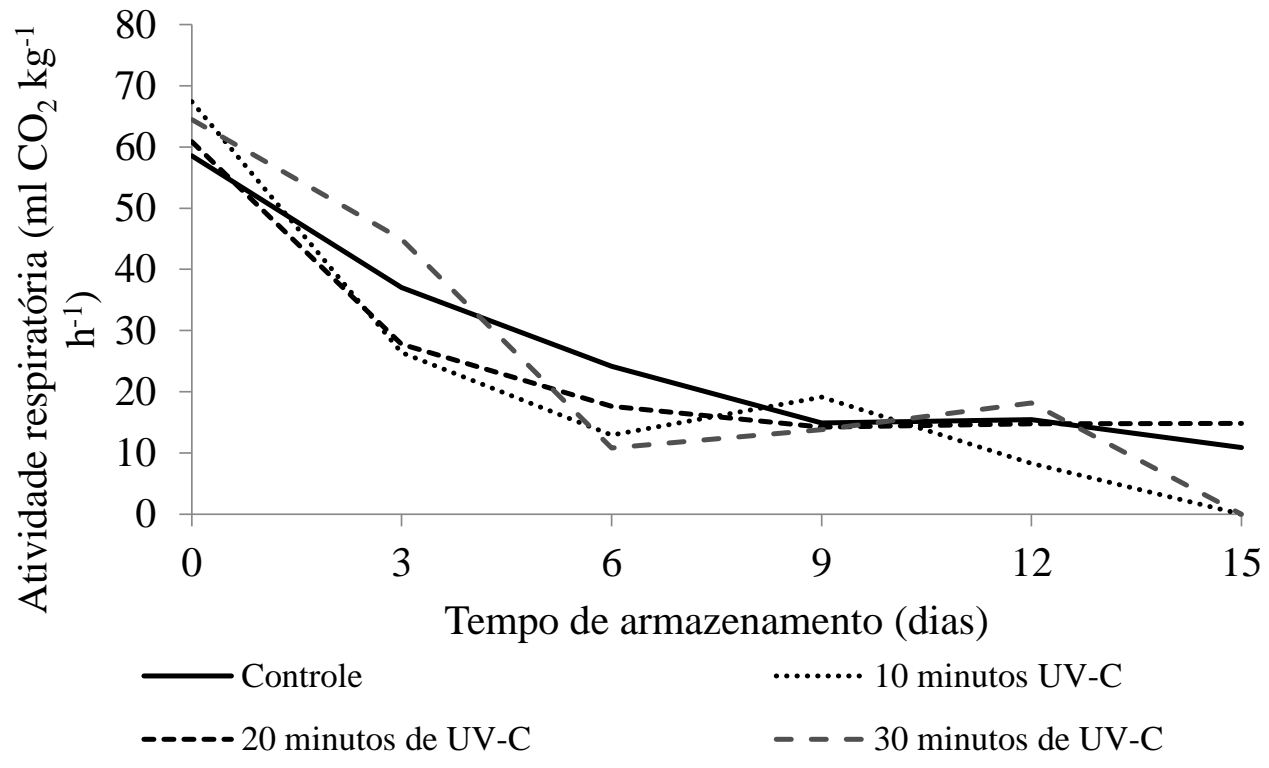

Para pH não houve diferença para tempo e tratamento nem para a interação, apresentando média geral de 3,52. Essa manutenção do $\mathrm{pH}$ durante o período de armazenamento é positiva, já que o $\mathrm{pH}$ é indicativo de deteriorização (CHITARRA; CHITARRA, 2007). De maneira geral podemos constatar que a radiação UV-C não influenciou no $\mathrm{pH}$ das lichias. Um tratamento de imersão sequencial, incluindo hipoclorito de sódio $(0,2 \%$, $\left.4 \mathrm{~min}, 52{ }^{\circ} \mathrm{C}\right)$, metabissulfito de potássio $(3 \%, 30$ min, $\left.26^{\circ} \mathrm{C}\right)$ e ácido clorídrico $(0,25 \mathrm{~N})$ contendo ácido ascórbico $\left(2 \%, 10 \mathrm{~min}, 26^{\circ} \mathrm{C}\right)$, seguido de irradiação gama, também não influenciou nos valores de $\mathrm{pH}$ da lichia 'Shahi' (KUMAR et al., 2012).

Sanches et al. (2017) também não verificaram influência dos tratamentos de radiação UV-C em tangerinas e tempo de armazenamento com o pH. A irradiação em morangos, assim como o período de armazenamento de 29 dias, também não interferiu no pH (FRANÇOSO et al., 2008). Já em morangos tratados com UV-C foram observados maiores valores de $\mathrm{pH}$ em relação ao controle ao final de dois dias de armazenamento (PAN et al., 2004).

A acidez apresentou diferença para o tratamento ( $p=0,0048$, Figura 3 ) e para tempo de armazenamento ( $p=0,0264$, Figura 4). Em todos os tempos de exposição à radiação os teores de acidez titulável apresentaram-se menores em relação ao controle. Em estudo com suco de uva integral verificou-se que o tratamento UV-C nas uvas provoca um estresse abiótico nas frutas, afetando a acidez titulável e relação sólidos/acidez titulável (HENRIQUE et al., 2016). 
Figura 3. Acidez titulável (g ácido málico $100 \mathrm{~g}^{-1}$ ) de lichias cv. Bengal submetidas a exposição da luz UV-C em armazenamento refrigerado ( $5 \pm 0,5 \stackrel{\circ}{ } \mathrm{C}$ e $90 \pm 5 \%$ UR) por 15 dias.

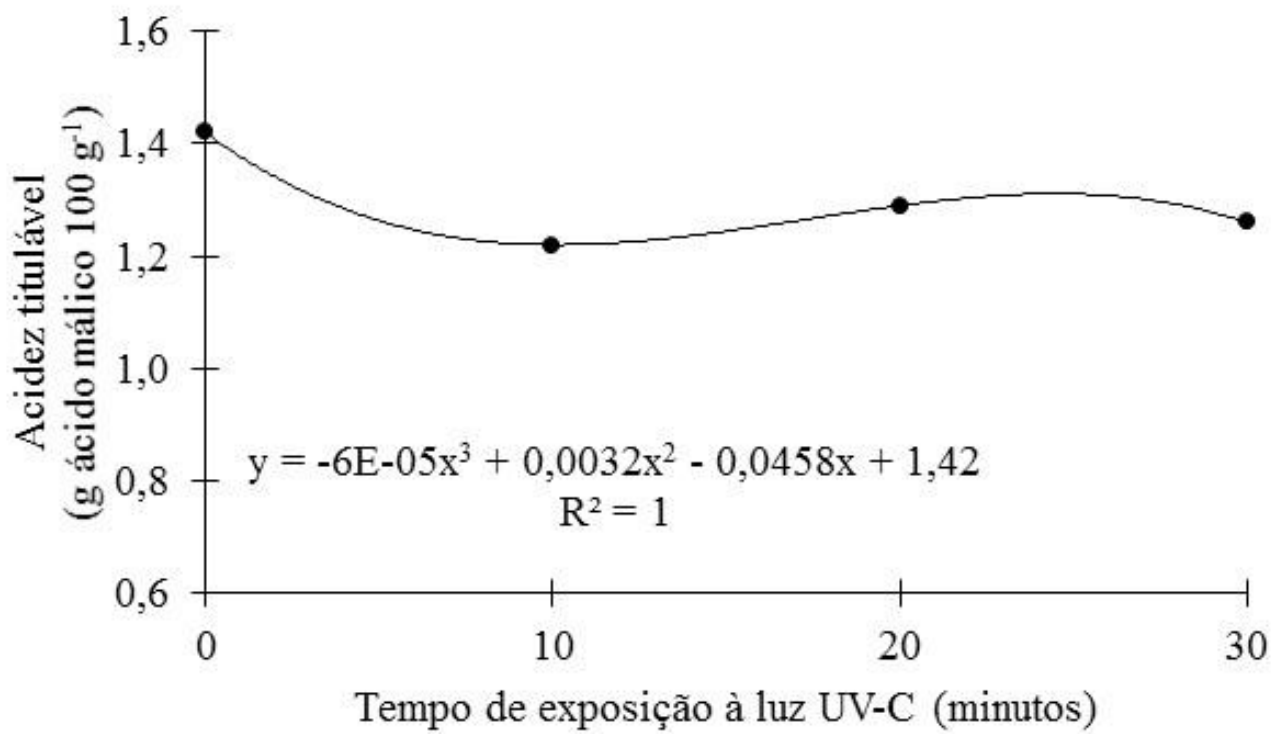

A acidez tende a diminuir com o tempo já os ácidos orgânicos são substratos no processo respiratório (CHITARRA; CHITARRA, 2007). As lichias apresentaram oscilações no teor de acidez titulável, porém sem diminuição no último dia de armazenamento. Marques (2015) encontrou menor acidez titulável em lichias $(0,63 \mathrm{~g}$ ácido málico $\left.100 \mathrm{~g}^{-1}\right)$ do que o presente trabalho $(1,22 \mathrm{~g}$ ácido málico $\left.100 \mathrm{~g}^{-1}\right)$.

Figura 4. Acidez titulável ( $\mathrm{g}$ ácido málico $100 \mathrm{~g}^{-1}$ ) de lichias $\mathrm{cv}$. Bengal em função do período de armazenamento refrigerado $(5 \pm 0,5 \stackrel{\circ}{ } \mathrm{C}$ e $90 \pm 5 \%$ UR) por 15 dias.

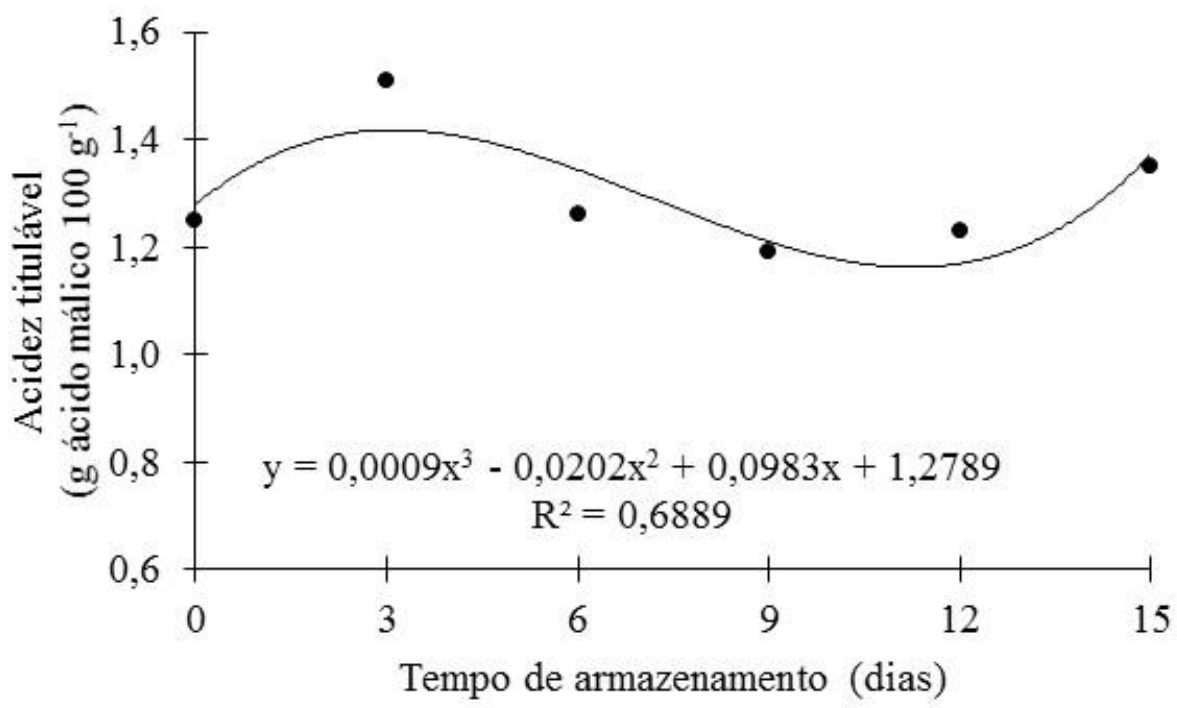

O teor de sólidos solúveis nos fornece um indicativo da quantidade de açúcares presentes na fruta. Normalmente esse teor aumenta com o período de armazenamento devido à maturação da fruta (FRANÇOSO et al., 2008). No presente trabalho não houve diferença com período de armazenamento e na interação dos fatores estudados, visto que a lichia é um fruto não climatérico.
Os sólidos solúveis foram significativos apenas em função dos tratamentos $(p=0,0184)$, sendo que houve declínio do teor em relação ao aumento da dose de radiação ultravioleta (Figura 5), possivelmente devido a maior degradação via glicólise com aumento da respiração e/ou concentração desses solutos devido a perda de massa, resultado semelhante com tangerinas à exposição UV-C, em que, exceto no dia 0 , todos 
os dias as frutas sem UV-C apresentavam maiores valores de sólidos solúveis (SANCHES et al., 2017).

Sem a exposição à luz UV-C, a lichia apresentou 19,10 ${ }^{\circ}$ Brix, teor próximo do encontrado por Jiang et al. (2004) $\left(15,7^{\circ}\right.$ Brix) e acima do encontrado por Marques (2015) $\left(6,25^{\circ}\right.$ Brix).

Figura 5. Sólidos solúveis ( ${ }^{\circ}$ Brix) de lichias cv. Bengal em função dos tratamentos de exposição a luz UV-C

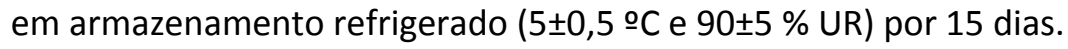

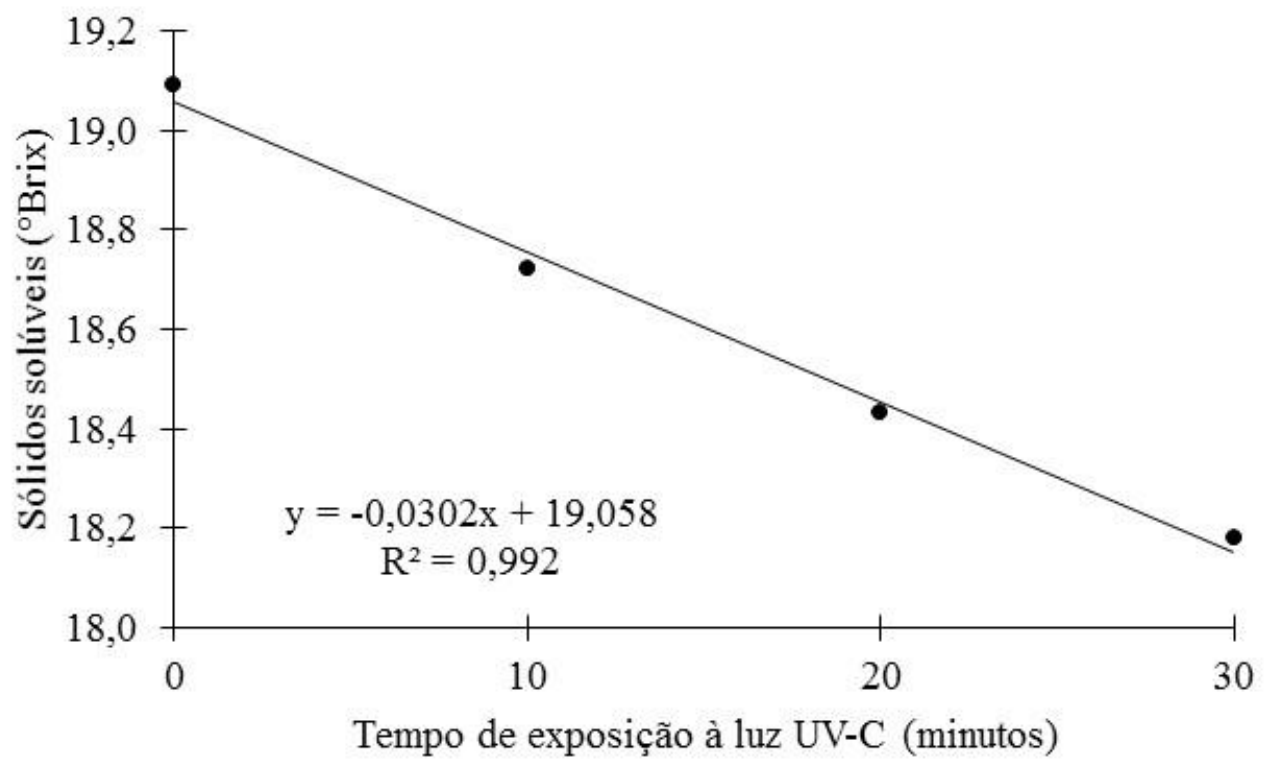

Para "Ratio" houve diferença em função dos tratamentos $(p=0,0037$, Figura 6) e em função do tempo ( $p=0,0333$, Figura 7$)$. O ratio, relação de sólidos solúveis e acidez titulável, é um parâmetro que indica qualidade do fruto (CHITARRA; CHITARRA, 2007). Os frutos do controle apresentaram menor índice de maturação $(13,8)$ quando comparados aos frutos tratados $(16,1 ; 15,2 ; 14,8)$, evidenciando que esses frutos estão melhores conservados.

Figura 6. "Ratio" (índice de maturação) em lichias cv. Bengal em função dos tratamentos de exposição a luz

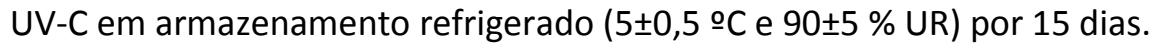

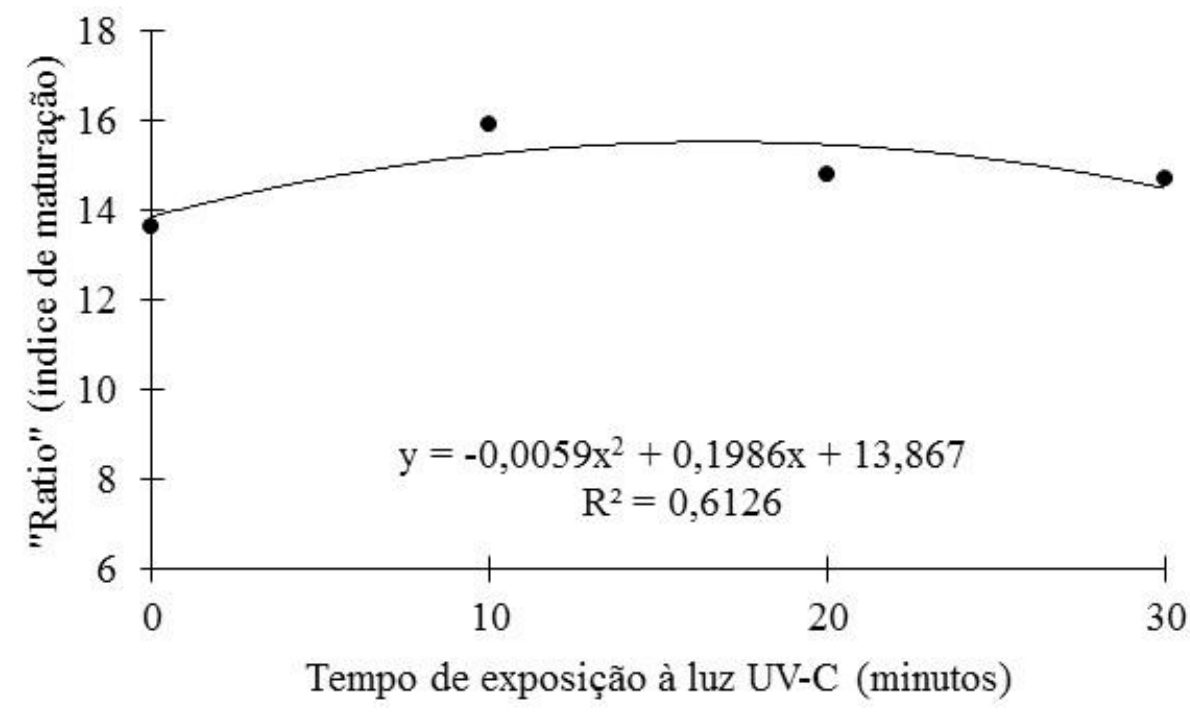


Verificou-se relação inversa com a acidez titulável durante os dias de armazenamento, aumento até o décimo segundo dia e posteriormente redução até os 15 dias. Não foi verificada alterações no "ratio" de uvas 'Niagara
Rosada' submetidas à diferentes doses de radiação UV-C (CIA et al., 2009), diferente ao presente trabalho.

Figura 7. "Ratio" (índice de maturação) de lichias cv. Bengal em função do tempo de armazenamento sob

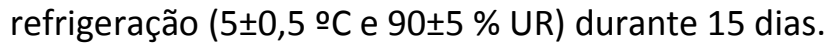

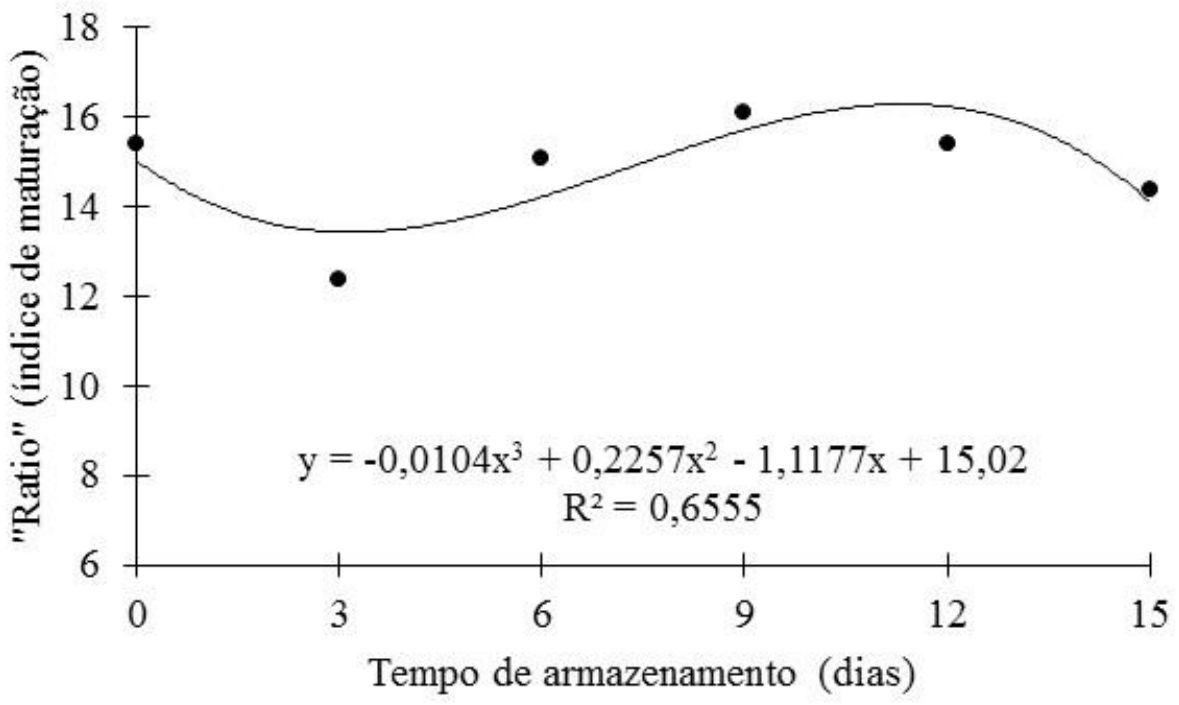

Para ácido ascórbico houve interação entre os fatores $(p=0,0243)$ (Figura 8). 0 tratamento controle e o a exposição de UV-C por 10 minutos tiveram diminuição do teor de ácido ascórbico durante os 15 dias de armazenamento. Enquanto que as lichias submetidas à 20 e 30 minutos de exposição à radiação UV-C mantiveram a concentração de ácido ascórbico durante $o$ período de armazenamento refrigerado. Resultado semelhante foi encontrado por Sanches et al. (2017), em que o controle e o tratamento com menor exposição à radiação UV-C tiveram maiores quedas do que os frutos com maior tempo de exposição durante 25 dias de armazenamento.

Essa diminuição do ácido ascórbico com o período de armazenamento nos tratamentos de 20 e 30 minutos pode ser explicada pela oxidação do ácido ascórbico para ácido dehidroascórbico (BARRETT; SOMOGY; RAMASWAMY, 2005), já o processo de irradiação causa essa oxidação que não afeta a qualidade nutricional (MOLINS, 2001). 
Figura 8. Ácido ascórbico (mg $\left.100 \mathrm{~g}^{-1}\right)$ em lichias cv. Bengal submetidas a exposição da luz UV-C em função do período de armazenamento sob refrigeração ( $5 \pm 0,5$ 드 e $90 \pm 5 \%$ UR) durante 15 dias.

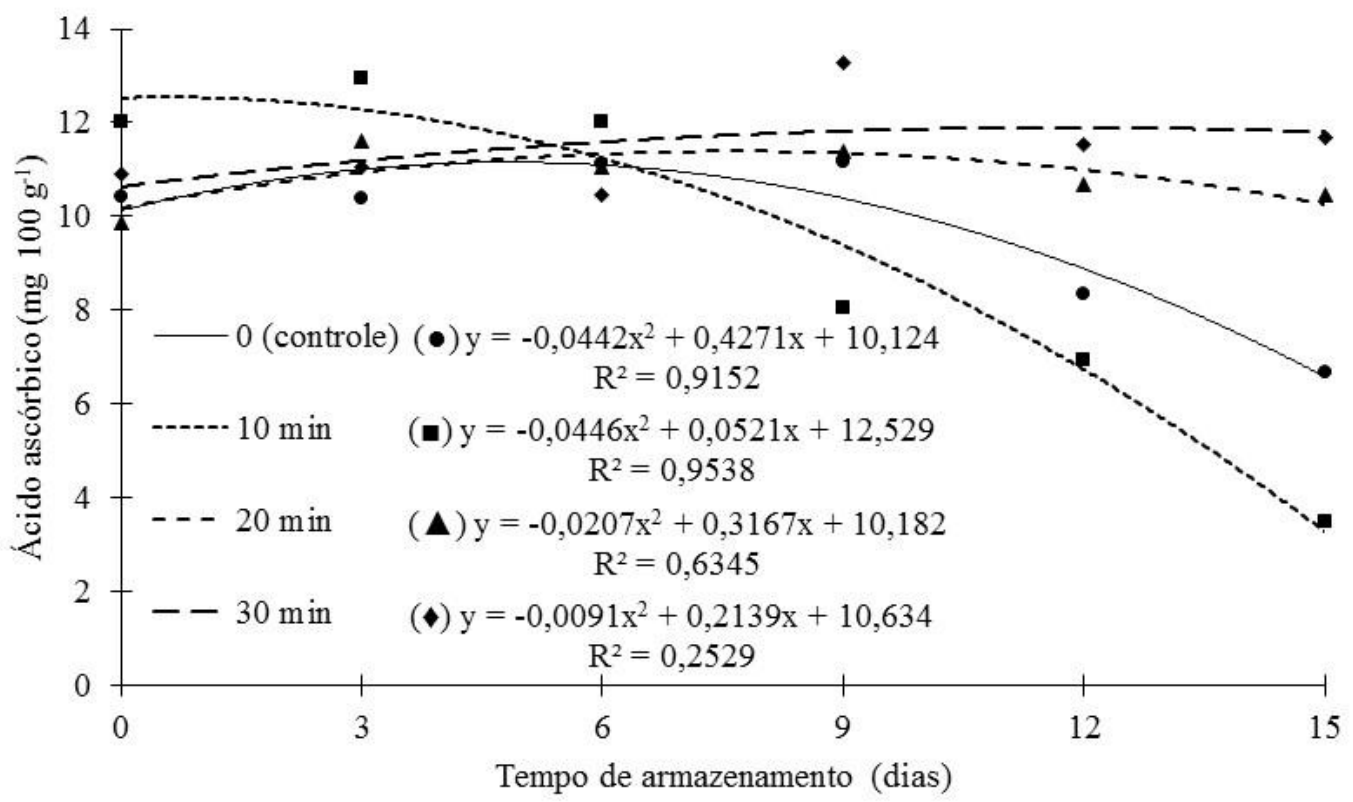

\section{Conclusões}

A radiação UV-C não foi efetiva para a conservação pós-colheita de lichias 'Bengal' em armazenamento refrigerado $(5 \pm 0,5 \stackrel{\circ}{\circ}$ e $90 \pm 5 \%$ UR) por 15 dias.

Os tratamentos de 20 e 30 minutos de exposição à radiação UV-C foram eficazes na manutenção da concentração de ácido ascórbico.

\section{Referências:}

AGUILA, J. S. D.; HEIFFIG-AGUILA, L. S.; SASAKI, F. F.; ORTEGA, E. M. M.; KLUGE, R. A. Efeito de antioxidantes na taxa respiratória e na produção de etileno de lichia 'bengal' armazenada sob refrigeração. Revista Iberoamericana de Tecnología Postcosecha, Hermosillo, v. 10, n. 1, p. 8-13, 2009.

BARRETT, D. M.; SOMOGY, L.; RAMASWAMY, H. Processing in fruit. Science and technology. 2. Ed. Boca Raton: CRC Press, 2005. p. 221-260.

BRASIL. Ministério da Sáude. Instituto Adolfo Lutz. Métodos físicos e químicos para análise de alimentos. 4. ed. São Paulo: Instituto Adolfo Lutz, 2008. 1020 p.

BLEINROTH, E.W.; ZUCHINI, A.G.; POMPEU, R.M. Determinação das características físicas e mecânicas de variedades de abacates e sua conservação pelo frio. Coletânea do Ital, Campinas, v.7, n.1, p.29-81, 1976.
CAMPOS, A.J.; VIEIES, R.L.; NEVES, L.C.; ROBLES, P.A.; CALERO, F.A. Ultraviolet radiation (UV-C) in characterization postharvest of tomato 'Pitenza'. Revista. Ibero Americana 10 de. Tecnología Postcosecha, México, v.12, n.2, p.192-198, 2011.

DAIUTO, E. R. Pós colheita do abacate 'Hass' submetido a radiação UV-C. Revista Colombiana de Ciências Hortícolas, Bogotá, v.7, n.2, p.149160 ,

2013.

https://doi.org/10.17584/rcch.2013v7i2.2231

DEL AGUILA, J. S.; HOFMANLL, P.; CAMPBELL, T.; MARQUES, J. R.; HEIFFIG- DEL AGUILA, L. S.; KLUGE, R. A. Pré-resfriamento em água de lichia 'B3' mantida em armazenamento refrigerado. Ciência Rural, Santa Maria, v. 39, n. 8, p. 23732379, 2009. https://doi.org/10.1590/S010384782009000800016

FRANÇOSO, I. L.; COUTO, M. A. L.; CANNIATTIBRAZACA, S. G.; ARTHUR, V. Alterações físicoquímicas em morangos (Fragaria anassa Duch.) irradiados e armazenados. Ciência e Tecnologia de Alimentos, Campinas, v.28, n.3, p. 614-619, jul.-set. 2008. https://doi.org/10.1590/S010120612008000300017

HASLER, C. M.; BLOCH, A. S.; THOMSON, C. A.; ENRIONE, E.; MANNING, C. Position of the American Dietetic Association: Functional foods. Journal of the American Dietetic Association, v. 
104, n.5, p.814-26, 2004. https://doi.org/10.1016/j.jada.2004.03.015

HENRIQUE, P. C. et al. Coloração, parâmetros físico-químicos e potencial antioxidante de sucos de uva integrais submetidos a diferentes doses de radiação UV-C. Ciência e agrotecnologia, v.40, n.2, p.226-234, 2016.

HUANG, X.M. Lychee production in China. In: Papademetriou, M. K.; Dent, F. J. (Eds.). Lychee Production in the Asia-Pacific Region. Food and Agricultural Organization of the United Nations, Bangkok, Thailand, 2002. p. 41-54.

JIANG, Y.; LI, Y.; LI, J. Browning control, shelf life extension and quality maintenance of frozen litchi fruit by hydrochloric acid. Journal of Food Engineering, n.63, p.147-151, 2004. https://doi.org/10.1016/\$0260-8774(03)00293-0

KHADEMI, O.; ZAMANI, Z.; POOR AHMADI, E.; KALNTARI, S. Effect of UV-C radiation on postharvest physiology of persimmon fruit (Diospyros kaki Thunb.) cv. 'Karaj' during storage at cold temperature. International Food Research Journal, Selangor, v. 20, n.1, p.247-253, 2013.

KUMAR, S. et al. Inhibition of Pericarp Browning and Shelf Life Extension of Litchi by Combination Dip Treatment and Radiation Processing. Food Chemistry, Barking, v. 131 , n. 4, p. 1223-1232, 2012.

https://doi.org/10.1016/j.foodchem.2011.09.108

LEE, S. K.; KADER, A. A. Preharvest and postharvest factors influencing vitamin $C$ content of horticultural crops. Postharvest Biology and Technology, v.20, p.207-220, 2000. https://doi.org/10.1016/S0925-5214(00)00133-2

LIMA, F. V.; AGUILA, J. S. D.; ORTEGA, E. M. M.; KLUGE, R. A. Pós-colheita de lichia 'Bengal' tratada com etileno e 1-metilciclopropeno. Ciência Rural, Santa Maria, v.41, n.7, p.11431149, 2011. https://doi.org/10.1590/S010384782011005000095

LIMA, R. A. Z.; ABREU, C. M. P.; ASMAR, S. A.; CORRÊA, A. D.; SANTOS, C. D. Embalagens e recobrimento em lichias (Litchi chinensis Sonn.) armazenadas sob condições não controladas.
Ciência e Agrotecnologia, Lavras, v. 34, n.4, p. 914-921, 2010.

LU, J. Y.; LUKOMBO, S. M.; STEVENS, C.; KHAN, V. A.; WILSON, C. L.; PUSEY, P. L.; CHAULTZ, E. LoW dose UV and gamma radiation on storage rot and physicochemical changes in peaches. Journal Food Quality, v.16, n.1, p.301-309, 1993. https://doi.org/10.1111/j.1745-

4557.1993.tb00115.x

MARQUENIE, D.; MICHELS, C. W.; IMPE, J. F., SCHREVENS, E.; NICOLAI, B. N. Pulsed white light in combination with (UV-C) and heat to reduce storage rot of strawberry. Postharvest Biology and Technology, Amsterdam, v.28, p.455-461, $2003 . \quad$ https://doi.org/10.1016/S09255214(02)00214-4

MARQUES, D. Efeitos da radiação gama na conservação e características físico-químicas de lichia (Litchi chinensis Sonn.). 2015. 56 f. Tese (Doutorado) - Universidade de São Paulo, 2015.

MENZEL, C. M.; WAITE, G. K. Litchi and Longan: botany, cultivation and uses. Queensland, Australia: CABI Publishing, 2005. 305 p.

MERKEN, H. M.; BEECHER, G. R. Measurement of foods flavonoids by high-performance liquid chromatography: a review. Journal of Agriculture and Food Chemistry, v. 48, n. 3, p. 577-597, 2000.

MOLINS, R. A. Food irradiation: principles and applications. New York: John Wiley \& Son, 2001. $488 \mathrm{p}$.

MOTTA, E. L. Avaliação da composição nutricional e atividade antioxidante de Litchi chinensis Sonn. ("Lichia”) cultivada no Brasil. 2009. Dissertação (Mestrado) - Universidade Federal do Rio de Janeiro, 2009.

PAN, J.; VICENTE, A. R.; MARTÍNEZ, G. A.; CHAVES, A. R.; CIVELLO, P. M. Combined use of UV-C irradiation and heat treatment to improve postharvest life of strawberry fruit, Journal of the Science of Food and Agriculture, v.84, p.1831-1838, 2004.

SANCHES, A. G.; SILVA, M. B.; MOREIRA, E. G. S.; COSME, S. S.; CORDEIRO, C. A. M.. Radiação uv-c na longevidade pós-colheita de tangerinas sob 
refrigeração, Revista Agrarian, Dourados, v.10, n.36, p. 129-135, 2017.

SARNI-MANCHADO, P.; LE ROUX, E.; LE GUERNEVÉ, C.; LOZANO, Y.; CHEYNIER, V. Phenolic composition of litchi fruit pericarp. Journal of Agriculture and Food Chemistry, v. 48, p. 5995-6002, 2000.

SOUZA, V. A.; VIEITES, L. R.; LIMA, P. P. G. Influência do tratamento térmico na qualidade de lichias refrigeradas. Revista ibero-americana de Tecnologia Postcosecha, Hermosillo, v. 10, n. 2, p. 110-119, 2010.

SOUZA, A. V.; VIEITES, R. L.; KOHATSU, D. S.; LIMA, G. P. P. Tratamento térmico na manutenção da coloração de lichias. Revista Brasileira de Fruticultura, v. 32, n. 1, p. 067-073, 2010.

SRICASTAVA, M.P.; SHARNA, N. UV-C Light as an effective physical method to control postharvest diseases. Journal of Biological and Chemical. Research, v.30, n. 2, p. 354-366, 2013.

STEVENS, C. et al. The effects of low-dose ultraviolet light- $C$ treatment on polygalacturonase activity, delay ripening and Rhizopus soft rot development of tomatoes. Crop Protection, Guildford, v. 23, p. 551 - 554, 2004.

VICENTE, A.R. et al. UV-C treatments reduce decay, keep quality and alleviate chilling injury in pepper. Postharvest Biology and Technology, Amsterdam, v.35, p.69-78, 2005.

WALL, M. M. Ascorbic acid and mineral composition of longan (Dimocarpus longan), lychee (Litchi chinensis) and rambutan (Nephelium lappaceum) cultivars grown in Hawaii. Journal of Food Composition and Analysis, v. 19, n. 6-7, p. 655-663, 2006.

WILSON, C. L.; EL-GHAOUTH, A.; CHALUTZ, E.; DROBY, S.; STEVENS, C.; LU, J. Y.; KHAN, V.; ARUL, $J$. Potential of induced resistance to control postharvest disease of fruits and vegetables. Plant Disease, v. 78, p. 837-843, 1994. 